\title{
Author Correction: Clear and transparent nanocrystals for infrared-responsive carrier transfer
}

\author{
Masanori Sakamoto (1) ${ }^{1}$, Tokuhisa Kawawaki (1) 1, Masato Kimura2, Taizo Yoshinaga ${ }^{3}$, Junie Jhon M. Vequizo (D) ${ }^{4}$, \\ Hironori Matsunaga4, Chandana Sampath Kumara Ranasinghe (i] ${ }^{4}$, Akira Yamakata (1) 4, Hiroyuki Matsuzaki (D) ${ }^{5}$, \\ Akihiro Furube $^{6} \&$ Toshiharu Teranishi (i) ${ }^{1}$
}

Correction to: Nature Communications https://doi.org/10.1038/s41467-018-08226-2, published online 24 January 2019

The original version of this Article omitted the fourth author Taizo Yoshinaga, who is from the 'Graduate School of Pure and Applied Sciences, University of Tsukuba, 1-1-1 Tennodai, Tsukuba, Japan'. Consequently, the third sentence of the Author Contributions, 'M.S. and M.K. synthesized the ITO NCs and ITO/semiconductor oxides' was revised to 'M.S., M.K. and T.Y. synthesized the ITO NCs and ITO/semiconductor oxides'. This has been corrected in both the PDF and HTML versions of the Article.

Published online: 17 April 2019

\begin{abstract}
(c) (i) Open Access This article is licensed under a Creative Commons Attribution 4.0 International License, which permits use, sharing, adaptation, distribution and reproduction in any medium or format, as long as you give appropriate credit to the original author(s) and the source, provide a link to the Creative Commons license, and indicate if changes were made. The images or other third party material in this article are included in the article's Creative Commons license, unless indicated otherwise in a credit line to the material. If material is not included in the article's Creative Commons license and your intended use is not permitted by statutory regulation or exceeds the permitted use, you will need to obtain permission directly from the copyright holder. To view a copy of this license, visit http://creativecommons.org/licenses/by/4.0/.
\end{abstract}

(C) The Author(s) 2019

\footnotetext{
${ }^{1}$ Institute for Chemical Research, Kyoto University, Gokasho, Uji, Kyoto 611-0011, Japan. ${ }^{2}$ Department of Chemistry, Graduate School of Science, Kyoto University, Gokasho, Uji, Kyoto 611-0011, Japan. ${ }^{3}$ Graduate School of Pure and Applied Sciences, University of Tsukuba, 1-1-1 Tennodai, Tsukuba, Japan. ${ }^{4}$ Graduate School of Engineering, Toyota Technological Institute, 2-12-1 Hisakata, Tempaku, Nagoya 468-8511, Japan. ${ }^{5}$ National Institute of Advanced Industrial Science and Technology (AIST), Tsukuba Central 2, 1-1-1 Umezono, Tsukuba, Ibaraki 305-8568, Japan. ${ }^{6}$ Department of Optical Science, Tokushima University, 2-1, Minamijosanjima-cho, Tokushima 770-8506, Japan. Correspondence and requests for materials should be addressed to M.S. (email: sakamoto@scl.kyoto-u.ac.jp) or to T.T. (email: teranisi@scl.kyoto-u.ac.jp)
} 